\title{
A Study for Implementation of Density Measurement Equipment for Asphalt Pavement based on the electromagnetic capacitance
}

\author{
Young-Ho Park, Gun-Kyun Kim, Jeong-Keun Nor, Jae-Kwon Ha \\ Bluewavetel Co., Ltd., Daejeon, 305-340, KOREA
}

\begin{abstract}
In this paper we developed density measurement equipment for Asphalt Pavement based on the electromagnetic capacitance. This kind of NonNuclear Density Gauges technology and products is used or studied in USA, Finland, Sweden as standardization of authorized method for pavement density measurement. Effective permitivity of pavement asphalt is characterized in electromagnetic capacitance by the asphalt material, mixed ratio, and harden grade of pavement asphalt. We can get a density value of asphalt by replacing value of electromagnetic capacitance with standard density value and characteristic transformation curve. We are conformed that measurement data according to temperature, humidity, and real field asphalt of our density measurement equipment can be a precise value.
\end{abstract}

Keywords: density measurement equipment

\section{INTRODUCTION}

The most general methods of density measurement equipment for asphalt pavement are Nuclear Density Gauges, GPR(Ground penetrating Radar) and Non-Nuclear Density Gauges technology based on dielectric pole and electromagnetic capacitance. The products of Non-Nuclear Density Gauges were actively developed in USA, Finland, and Sweden and are studied through laboratory and field analysis[1]. In 1987, measurement test of asphalt mixture by GPR method in Sweden was made but the satisfactory result did not come out with the current technology. Although Road Radar Inc. in Finland in 1993 measured asphalt pavement by developing the product that the GPR technology was applied, it did not attain the satisfactory result. Thereafter, Texas transportation Institute in USA in 1994-1995 studied technology and products by GPR. In 1996, University of Oulu and Neste in Finland developed products, and came to adopt it as asphalt density measurement method through reliance evaluation in 1997. The USA got a patent for the method of density measurement[2]. Also, it seems that Transtech Systems Inc. developed PQI(Pavement Quality Indicator) of NonNuclear Density Gauges for the first time in 1998 and is trying to measure density[3]. In Korea, however, density measurement equipment through core extraction is still applied, and research paper on development of density measurement equipment is recently presented[4]. When the real field asphalt density is measured, the existing method has generally taken sample extraction method. However, the advanced countries have started using non-destructive method under consideration on time, economy, and efficiency. And they are standardizing

* Corresponding author. E-mail : pyh601@hanmail.net Manuscript received Nov. 18, 2010 ; accepted Dec. 20, 2010 non-destructive method. On account of this, this paper introduces density measurement equipment developed with non- destructive method.

\section{BASIC CONCEPT FOR DENSITY MEASUREMENT OF ASPHALT PAVEMENT}

\subsection{Configuration for Asphalt Pavement}

Asphalt pavement is composed of the configuration like $<$ Figure $1>[5]$. Abrasion layer and middle one are not always necessary but can optionally be used. In selecting heating asphalt mixture, weather condition, regional condition, regional division, traffic density division, numbers of lanes, material condition, finished thickness of one layer, and construction are taken into consideration. Mixture with fluidity for high traffic density road, mixture with flexibility and durability for low traffic density road, and mixture with abrasion resistance for snowy area road whose surface can be worn away because of tire chain to prevent car slipping in winter are used.

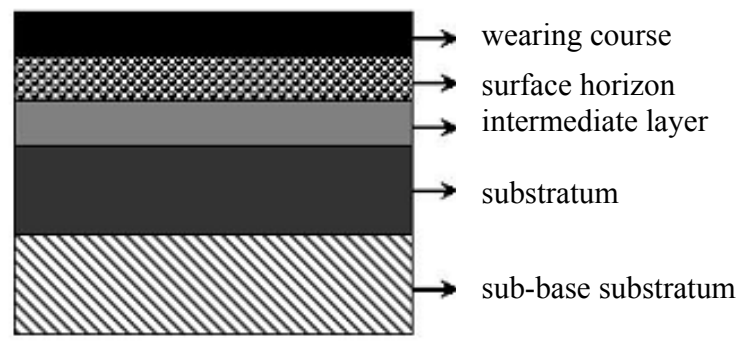

Sub-grade

Fig 1. Cont1guration of asphalt pavement 


\subsection{Basic Concept for density measurement of Asphalt}

Three layer Structure of Asphalt provides density-weight definition for unit volume of hardened materials, and appearance density can be formulated by dividing specimen volume into total weight. The maximum theoretical density for the given asphalt content is gained by dividing the weight of aggregate and asphalt by the volume that aggregate and asphalt occupy; however, the volume that the air occupies is not included. The maximum theoretical density is very important because it is used as a reference value to calculate various important properties including porosity.

\subsection{Model Interpretation for electromagnetic capacitance}

If asphalt mixture in a dry condition is composed of only asphalt binder \& rubble and each configuration rate is different, the electric characteristics can be modelized as $<$ Figure $2>$. It can be interpreted that two capacitors are serially combined in the configuration that two different permittivity with area $\mathrm{S}$ and distance $\mathrm{d} 1$, and $\mathrm{d} 2$ make up for. It is assumed that $\mathrm{d}$ is extremely small in comparison to the length size of ordinary plate. If permittivity of the first electromagnetic is $\varepsilon 1$, capacity becomes $\varepsilon_{1} \mathrm{~S} / \mathrm{d} 1$ and capacity of the second layer is $\varepsilon_{2} \mathrm{~S} / \mathrm{d} 2$. Because electromagnetic capacity $\mathrm{C}=\mathrm{Q} / \mathrm{V}$ is shown as charge \& electric potential difference, if one of them is supposed, the rest value can be gained.

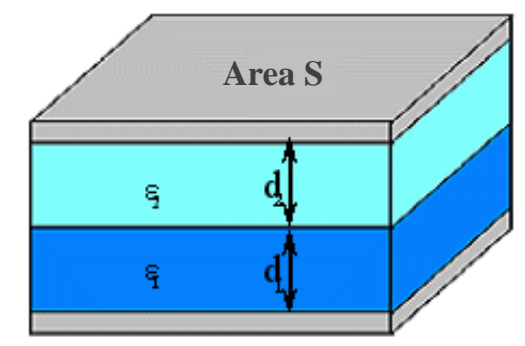

Fig 2. Model for different configuration rate

\section{IMPLEMENTATION OF DENSITY MEASUREMENT EQUIPMENT FOR ASPHALT PAVEMENT}

\subsection{Concept and Method for Implementation of Density Measurement Equipment}

Each layer composed of asphalt concrete mixture generates total non-permittivity change according to configuration volume ratio of mixture. Consequently, compacting density measurement equipment sees asphalt mixture as a dielectric;

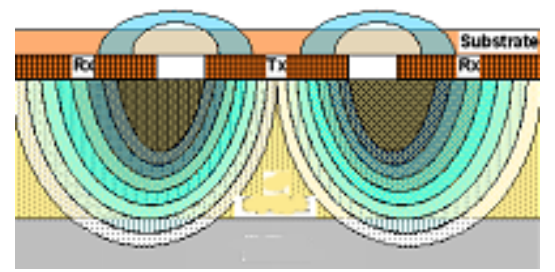

Fig 3. Electric field distribution of typical RF sensor antenna measures the level received by radiating always fixed levelelectromagnetic wave on the road surface; takes the method to convert level variations by dielectric changes into compacting density. When charge is forced on conductor, electromagnetic field is formed. If electromagnetic field is forced on dielectric, level value exposed on receiving plate by internal loss and dielectric value changes. By measuring this value, it is recognized that there would be asphalt mixture density changes or configuration component rate changes. $<$ Figure $3>$ shows electric field distribution of typical RF sensor antenna.

\subsection{Configuration for Implementation of Density Measurement Equipment}

$<$ Figure $4>$ is the development configuration chart of developed asphalt density measurement equipment. H/W consists of LCD Display, Keypad, Sensing circuit(Transmitting circuit, Receiving circuit), and sensing transmitting \& receiving antenna. $\mathrm{S} / \mathrm{W}$ is composed of signal analysis process and compensation algorithm that convert and keep received signal into density value. The micro processor for signal analysis process is used AVR 128 micro-controller[6].

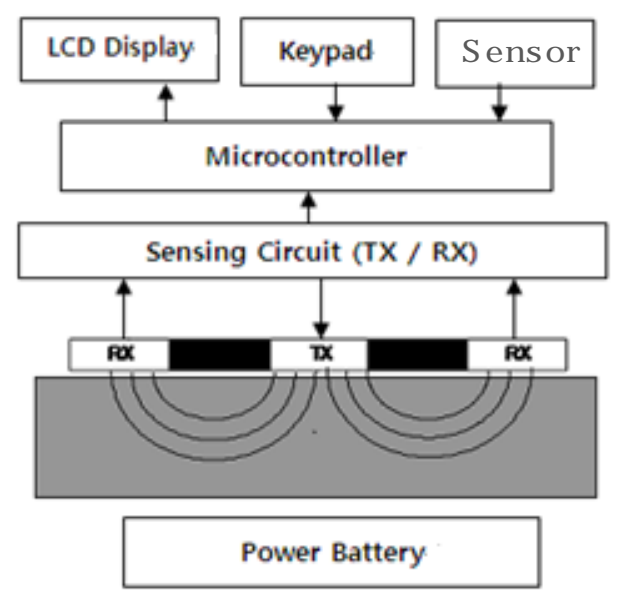

Fig 4. Implementation configuration

Sensing transmitting and receiving antenna device is adhered closely to asphalt mixture and case is grounded. If the even level high frequency voltage is forced through sensing receiving antenna, organic voltage level is perceived through receiving sensor antenna according to medium permittivity. This receiving signal level is input into ADC as a DC level through diode rectifier circuit and converted into digital level. Signal analysis process device converts density of measurement object by applying compensation algorithm appropriate to measurement environment and condition.

\subsection{Result Data for Implementation of Density Measurement Equipment}

Because asphalt density is less sensitive to temperature and more influential from humidity, accuracy of density measurement equipment is revealed by measuring temperature and humidity from the real field asphalt. 


\subsubsection{Result Data for Temperature}

First, it is confirmed that there is no big change in temperature and change only in medium as $<$ Table $1>$ shows measuring data as a result of measuring changes according to temperature.

Table 1. Measuring changes according to temperature

\begin{tabular}{|c|c|c|c|}
\hline material & Measuring time & $\begin{array}{l}\text { Measuring } \\
\text { temperature }\end{array}$ & $\begin{array}{l}\text { Density } \\
(\mathrm{Kg} / \mathrm{m} 3)\end{array}$ \\
\hline \multirow{3}{*}{$\begin{array}{c}\text { Parking } \\
\text { Area }\end{array}$} & $10: 10$ & 19.8 & 2400 \\
\hline & $15: 00$ & 32.9 & 2400 \\
\hline & $16: 00$ & 36.5 & 2400 \\
\hline \multirow{4}{*}{$\begin{array}{l}\text { office } \\
\text { floor }\end{array}$} & $10: 10$ & 31.0 & 4600 \\
\hline & $15: 00$ & 31.3 & 4610 \\
\hline & $16: 00$ & 30.8 & 4595 \\
\hline & $16: 30$ & 31.0 & 4600 \\
\hline \multirow{4}{*}{$\begin{array}{c}\text { Desk } \\
\text { (wood) }\end{array}$} & $10: 10$ & 18.0 & 1840 \\
\hline & $15: 00$ & 18.1 & 1850 \\
\hline & $16: 00$ & 18.3 & 1820 \\
\hline & $16: 30$ & 18.0 & 1860 \\
\hline \multirow{4}{*}{$\begin{array}{l}\text { Asphalt } \\
\text { sample }\end{array}$} & $10: 10$ & 20.0 & 2330 \\
\hline & $15: 00$ & 20.5 & 2300 \\
\hline & $16: 00$ & 19.8 & 2320 \\
\hline & $16: 30$ & 20.1 & 2340 \\
\hline
\end{tabular}

\subsubsection{Result Data for Humidity}

Second, it is confirmed that there is big change when humidity goes over $50 \%$ as $<$ Table $2>$ presents measuring data as a result of measuring humidity.

Table 2. Measuring changes according to humidity

\begin{tabular}{|c|c|c|}
\hline humidity & density $(\mathrm{Kg} / \mathrm{m} 3)$ & $\begin{array}{c}\text { Measuring } \\
\text { temperature }\end{array}$ \\
\hline \hline 10.7 & 2341 & $26^{\circ} \mathrm{C}$ \\
\hline 15 & 2360 & $24.4^{\circ} \mathrm{C}$ \\
\hline 26 & 2470 & $20.3^{\circ} \mathrm{C}$ \\
\hline 30 & 2490 & $20.8^{\circ} \mathrm{C}$ \\
\hline 41 & 2568 & $22^{\circ} \mathrm{C}$ \\
\hline 53.2 & 3165 & $23.5^{\circ} \mathrm{C}$ \\
\hline
\end{tabular}

\subsubsection{Result Data for Real Measurement on Asphalt Pavement}

Third, the predicted result could be obtained as a result of measuring the real field asphalt. Measuring density compensation of developed density measurement equipment referred to standard sample in $<$ Figure $5>$ of already known density value and used $2328 \mathrm{Kg} / \mathrm{m} 3$ of density.

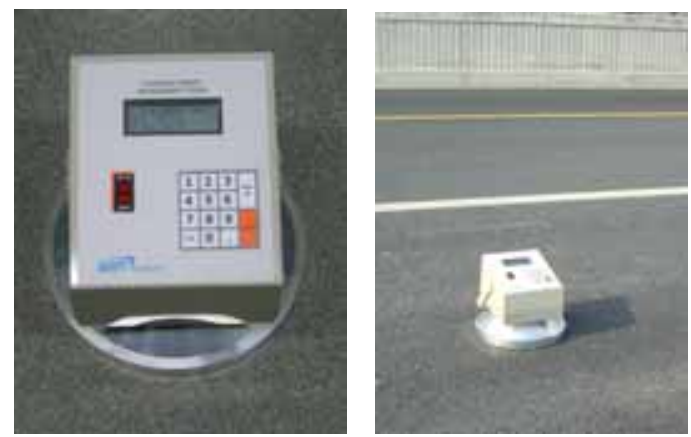

Fig 5. Density measurement equipment

It is decided that more precise density measurement would be possible by constructing various DB according to much more samples, thickness \& kind of materials, and mixing rate although developed density measurement equipment is measured from the standard of density value by standard sample.

Table 3. Measuring data for Asphalt density

\begin{tabular}{|c|c|c|}
\hline place & $\begin{array}{c}\text { Sample } \\
\text { density }(\mathrm{Kg} / \mathrm{m} 3)\end{array}$ & $\begin{array}{c}\text { Measure } \\
\text { density }(\mathrm{Kg} / \mathrm{m} 3)\end{array}$ \\
\hline Office floor & 2328 & $2300 \sim 2340$ \\
\hline Real field & real asphalt & $2330-2410$ \\
\hline
\end{tabular}

$<$ Table $3>$ shows measuring data for Asphalt density. Asphalt pavement density value is changed according to kinds of roads(local road, national road, and express way) and pavement execution date. As the result of measuring the real field asphalt density with developed density measurement equipment that measured the standard sample of $2328 \mathrm{Kg} / \mathrm{m} 3$ density and showed favorable result, the density of roads paved within 2 months among asphalt roads from Yuseong to Gasuwon in Daejeon was measured as $2330-2410 \mathrm{Kg} / \mathrm{m} 3$ according to their location; the density of asphalt mixture that many years had passed around Yuseong Homeplus in Daejeon was measured as $2530-2580 \mathrm{Kg} / \mathrm{m} 3$. It is, therefore, verified that the density of asphalt mixture is increasing as time passes. This case has already been reported in foreign countries.

\section{CONCLUSION}

The most vulnerable part of density measurement equipment by electromagnetic capacitance method is that density changes greatly according to dampness of measurement asphalt mixture. Accordingly, compensation algorithm by humidity needs to be applied because the exact measurement is difficult on the condition of more than $60 \%$ humidity. Research \& development of the standard with which non-destructive pavement density measurement method can officially be approved are required in Korea as well because asphalt density measurement and approval method by non-destructive method is adopted as standardized construction in other countries. 
The expected effects of developed asphalt density measurement equipment are as follows:

- Time $\&$ cost cut-down in examining road construction because asphalt density measurement can be done more conveniently.

- Efficiency enlargement according to the cut-down of construction supervision, management, and administrative affairs.

- Developed product can be utilized in the related fields such as army \& crime investigation, and underground excavation by making use of the function to detect construction field of objects laid underground.

- Efficiency enlargement from the cut-down of construction supervision \& management and administrative affairs.

It is considered that industry-academic cooperation business would be necessary to develop domestic standardization technology for process \& non-destructive density measurement because asphalt density measurement has already been adopted as standard method of construction in other countries. From now on, industry-academic cooperation is required for making data on basic density value for mixture characteristics, mixing rate, and thickness in order to enhance the accuracy of density measurement according to the thickness of asphalt or concrete. Non-destructive asphalt density measurement method is the one that can reduce time and cost in terms of road pavement and maintenance. Therefore, it is judged that Korea should study the standard for verifying road pavement and move introduction of road pavement density measurement method by non-destructive method.

\section{REFERENCE}

[1] Pedro Romero, Evaluation of Non -Nuclear Gauges to Measure Density of Hot-Mix Asphalt Pavements, master thesis, University of Utah, 2002.

[2] Robert A.Sovik, Richard N. Hosternman, George G. Moross, Paving Material Density Indicator and Method Using Capacitance. Patent No. US005900736, May 1999.

[3] Transtech System, Pavement Quality Indicator Model 301 Operator's Handbook, Transtech System Inc, New York, 2003.

[4] .K. Kim, J.K. Nor, J.K. Ha and Y.H. Park, "Study for density measurement in asphalt pavement" KIEES, Vol. 19, no 1, Nov. 2009, p. 225.

[5] Ministry of Construction \& Transportation, Direction for mixture design of heating asphalt, MOCT, Seoul, 2005.

[6] Bong-gil Song, AVR ATmega128 micro controller, Seongandang, Seoul, 2008.

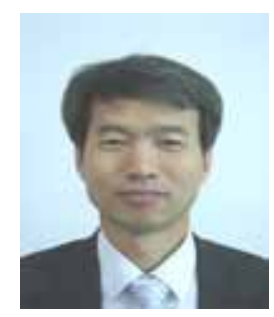

\section{Young-Ho Park}

$\mathrm{He}$ received the B.S degree in the department of Electronics Engineering from Seoul City University in 1983, and he received M.S degree in division of Electrical Engineering from University of Missouri at Columbia, USA in 1991. He worked for ETRI as a senior researcher and he is now director in Bluewavetel co., Ltd. His main research interests include microprocessor applications and communication system applications.

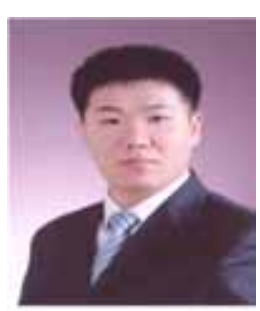

\section{Gun-Kyun Kim}

$\mathrm{He}$ received the B.S degree in the department of Electronics Engineering from DongSeo University in 2005, and he received M.S degree in division of Information System Engineering from DongSeo University in 2008. He is now engineer in BlueWaveTel co., Ltd. and his main interests include antenna and RF modules.

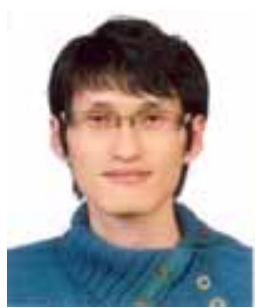

\section{Jeong-Keun Nor}

He received the B.S degree in the department of Electronics Engineering from HanBat national University in 2006. $\mathrm{He}$ is now engineer in BlueWaveTel co., Ltd. and his main interests include antenna and RF modules.

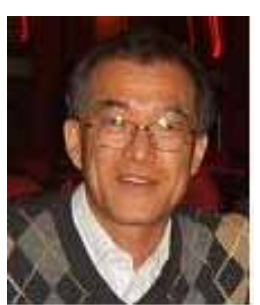

\section{Jae-Kwon Ha}

He received the B.S., M.S in electronics engineering from KyungPook national university, Korea in 1980, 1982 respectively and also received $\mathrm{Ph} . \mathrm{D}$. in electronics engineering from Chung Nam national university, Korea in 2003. Since then, he had been with various telecommunication system development center, ETRI. He established Bluewavetel co., Ltd., in 2000 June. He has developed high performance antenna and RF module. His main research interests include microprocessor applications and high-performance antenna and RF modules. 